Human Ethology Bulletin - Proc. of the V. ISHE Summer Institute (2016): 60-73

Research Article

\title{
DisguST AND FACIAL EXPRESSION RECOGNITION ACROSS THE Menstrual CyCle
}

\author{
Ana Mikolić \\ Department of Psychology, Faculty of Humanities and Social Sciences, University of \\ Zagreb, Croatia \\ ana.mikolic153@gmail.com
}

\begin{abstract}
In the luteal phase of the menstrual cycle, immune responses decrease under the influence of progesterone. This immunosuppression facilitates embryo implantation and maintenance of pregnancy, but at the same time it leads to a greater vulnerability to diseases. The aim of this study was to investigate whether the prophylactic compensatory behaviours, which could decrease the probability of disease, were more expressed in the luteal phase. To this end, disgust sensitivity and recognition of disgusted facial expressions were assessed. The participants had to rate how dangerous and disgusting they found the neutral images and those related to contagion and contamination. Speed and accuracy of labelling facial expressions of disgust and other basic emotions were also measured. The data of $N=40$ participants were analysed, which were previously collected two times, first during their early follicular and then during luteal phases of the cycle. The compensatory prophylaxis hypothesis was not confirmed: no differences in disgust and danger ratings were observed. The facial expression of disgust was not recognized more quickly and accurately during the luteal phase. However, in line with other studies that indicate changes in social cognition across the menstrual cycle, it was found that basic emotions are recognized more accurately in the early follicular phase compared to the mid-luteal phase.
\end{abstract}

Keywords: Menstrual cycle, vulnerability to disease, disgust sensitivity, pathogens, recognition of facial expressions. 


\section{INTRODUCTION}

Throughout the entire human history, as well as in modern times, pathogens have been ubiquitous. Their harmful effects on survival and inclusive fitness constituted selection pressure for developing protective mechanisms - physiological barriers, a complex immune system, but also a "behavioural immune system" (Curtis, de Barra, \& Aunger, 2011; Schaller $\&$ Duncan, 2007). The behavioural immune system includes a) detection of potential infectious pathogens in the environment; b) emotional and cognitive responses to them; and c) pathogens-avoidance behaviours. The perceptual stimuli which activate the system are a broad class of cues superficially related to infectious disease, and like every other selfprotection system, this one also includes false-positive errors (Schaller \& Duncan, 2007). Moreover, the behavioural immune system involves certain losses, such as the loss of time and energy that could otherwise be spent on important social interactions. Therefore, it is characterized by functional flexibility (Schaller, Park, \& Kenrick, 2007): under the circumstances in which people are more vulnerable to diseases, or they perceive themselves to be, they are more sensitive to cues of pathogens and have stronger aversive reactions. For instance, the perceived threat of contagion is associated with stronger prejudices towards obese, older and disabled people (Duncan \& Schaller, 2009; Park, Faulkner \& Schaller, 2003; Park, Schalle \& Crandall, 2007), as well as with a temporary inhibition of social interactions (Mortensen, Becker, Ackerman, Neuberg \& Kenrick, 2010). Furthermore, individuals who perceive themselves more vulnerable to infections show stronger preferences for cues of health on faces, but not for other features they find attractive (Welling, Conway, DeBruine \& Jones, 2007).

Besides infectious disease outbreaks, health status and some personality traits, there are some other factors that can make individuals more attentive to possible health threats. One of them is the menstrual cycle.

\section{Menstrual cycle}

The menstrual cycle consists of five phases: menstrual, follicular, ovulation, luteal and premenstrual (McFarlane, Martin \& Williams, 1988). Different phases are characterized by different levels of gonadotropins, estrogens and progesterone. Estrogens are important for the development of sex organs, pregnancy and its maintenance. To enable pregnancy, the mother's body has to tolerate the presence of a half-foreign blastocyst, namely the father's genetic material. Since the main task of the immune system is to detect and attack foreign bodies, immune responses are decreased by progesterone in the luteal phase of the cycle and later in pregnancy. These changes represent an adaptation that protects the blastocyst from the maternal immune system and facilitates its reception and development (Fleischman \& Fessler, 2010). However, the cost of this adaptation is heightened susceptibility to diseases during the luteal phase and the first trimester of pregnancy. For example, the levels of cytokines decline and the number of natural killer cells is decreased (Faas et al., 2000; Trzonkowski et al., 2001). 
According to the functional flexibility hypothesis (Schaller, Park \& Kenrick, 2007), under these circumstances, women should recognize cues of pathogens more efficiently and respond to them more strongly. However, the effects of progesterone on prophylactic compensatory behaviour have not been sufficiently investigated yet. Jones et al. (2005) found stronger preferences for healthy faces with higher levels of progesterone. Navarette \& Fessler (2007) observed intergroup bias and more negative evaluations of outgroup members in the first trimester of pregnancy. Finally, immunosuppression should also affect the food selection and disgust sensitivity in the food domain, and the same patterns should be observed in other domains of pathogen-related disgust.

\section{Disgust}

Disgust is one of the basic emotions and it is associated with a universal facial expression (Ekman \& Friesen, 1975), avoidance behaviour, characteristic neuro-physiological changes and negative affect (Curtis, de Barra \& Aunger, 2011). Disgust elicitors seem to be culturally universal as well, such as bodily fluids, dirty environment, sick, dead or deformed people, spoiled and unknown food, some animals and sexual behaviours (Curtis \& Biran, 2001; Haidt, McCauley \& Rozin, 2007). According to the evolutionary framework, disgust is considered to be an adaptive system for disease avoidance (Curtis \& Biran, 2001). Moreover, the emotion of fear is also considered crucial for the behavioural immune system (Prokop, Usak \& Frančovičova, 2010), motivating avoidance in the presence of potential pathogens.

Despite this universality of disgust, there are some inter- and intra-individual differences in disgust reactions (Haidt, McCauley \& Rozin, 2007; Curtis, de Barra \& Aunger, 2011). Disgust sensitivity depends on personality traits, current state and learning abilities (Curtis, de Barra \& Aunger, 2011). In line with the compensatory prophylaxis hypothesis, Fleischman \& Fessler (2010) found the association of progesterone levels with bathroom behaviour, grooming, disgust sensitivity and obsessive-compulsive symptoms in the cleaning domain. Moreover, disgust sensitivity in the food domain seems to be heightened in the first trimester of pregnancy compared to the second and third (Fessler, Eng \& Navarette, 2005). However, not all research has shown disgust-related changes in periods of higher progesterone (Fleischmann \& Fessler, 2007; Fessler \& Navarette, 2003).

Conway (2007) found changes in the perception of facial expressions of fear and disgust as a function of progesterone levels. The facial expression of disgust could serve as a signal of a pathogen threat in the environment, and its efficient recognition on the faces of others could help us to protect ourselves from potential threats by avoidance and nonconsumption.

\section{Emotion recognition}

Many studies focus on cognitive, affective and behavioural changes across the menstrual cycle, but research on hormonal variations and emotion recognition is still sparse. In one study, negative correlation between the levels of progesterone and emotion recognition was 
found, and the participants "over-perceived" emotions of anger and disgust, mostly in angry and sad expressions, in the luteal phase of the cycle (Derntl, Kryspin-Exner, Fernbach, Moser \& Habel, 2008). However, more accurate recognition in the follicular phase compared to the luteal has not been consistently found for all basic emotions (Guapo et al., 2009). Furthermore, some researchers showed stronger amygdala response in the low progesterone phase (Derntl et al., 2008), while some observed stronger amygdala reactivity to angry and fearful faces after the progesterone administration (Van Wingen et al., 2007).

To conclude, the available findings indicate a more accurate emotion recognition in the follicular phase in comparison to the luteal phase, but there is still inconsistency and lack of knowledge regarding this topic. Therefore, one of the goals of this study was to further investigate facial expression recognition as a function of the menstrual cycle, with particular emphasis on disgust.

The main goal of this study was to investigate the presence of prophylactic compensatory mechanisms in the luteal phase of the menstrual cycle, which is characterized by reduction of immune responses and consequently by higher vulnerability to infectious diseases. Accordingly, it was predicted that women would show more aversive reactions to stimuli associated with contagion and contamination in the luteal phase of the cycle than in the early follicular. More precisely, in this period of greater susceptibility to diseases, higher disgust and danger ratings of disgusting objects were expected, as well as better and faster recognition of the disgusted expression. Overall, emotion recognition was expected to be more accurate in the follicular phase than in the luteal.

\section{METHODS}

\section{Participants}

85 naturally-cycling women, all non-pill users, participated in the study. However, due to irregularities of their menstrual cycles during the procedure, and, consequently, the conclusion that measurements maybe were not taken in the right period of the cycle (since there was no additional blood or saliva data), one portion of the participants was excluded from the analysis. The analysis was performed on $\mathrm{N}=40$ participants, with the average age of 22.9 years $(\mathrm{SD}= \pm 6.659)$ and average menstrual cycle length of 28.7 days $(\mathrm{SD}= \pm 2.444)$.

\section{Procedure}

The participants were recruited via the web page of the Department of Psychology of the University of Zagreb, Google Groups and Facebook. According to the within-subject design of the study, every participant took part in two experimental sessions. One was in the early follicular phase of the menstrual cycle (the first 6 days of the cycle) and the other in their mid-luteal phase ( 7 days before the next menstruation). The exact date was determined according to the first day of the last menstruation and the expected date of the next one. The order was rotated among the participants; therefore, half of them were first tested in their 
follicular phase, and the other half in the luteal phase. If the menstruation had started $7 \pm 2$ days after the luteal phase session, the testing was considered valid. The follicular phase testing was conducted during the first 6 days after the menstruation had started, thus a subsequent verification was not required. In the beginning of the study every participant would fill in the menstrual cycle questionnaire. During both sessions, the participants had to do two computer tasks programmed in E-prime v1.1 (Schneider, Eschman \& Zuccolotto, 2002): the Emotion Recognition Task and the Disgust Sensitivity Task (see below). In order to avoid practice effects, two versions of every task were used and their order was rotated among the participants and sessions. Thus, half of the participants had the first version in the luteal phase and half of them in the follicular phase. All photographs were presented in a randomized order.

The Emotion Recognition Task contained facial expressions of basic emotions (fear, sadness, happiness, surprise, disgust, anger) from the NimStim Set of Facial Expressions (Tottenham et al., 2009). For the purposes of this study 66 pictures were selected and split in two versions. They were balanced on gender and race of the actor, mouth opening (open vs. closed), and accuracy ratings known from the past research (Tottenham et al., 2009). Every version contained basic emotions, each shown by 3 female and 3 male actors, 3 times with open and 3 times with closed mouth (except for surprise which exists only in an openmouthed version). The task was to identify the emotion expressed on the presented photograph, using the keyboard numbers and selecting either one of the 6 basic emotions or the "nothing from above" option. Emotional expressions were presented randomly, one at the time. There was no time limit, but the participants were instructed to respond as quickly as possible. Both labelling speed and accuracy were registered.

The Disgust Sensitivity Task consisted of 44 photographs from the International Affective Picture System (IAPS; Lang, Bradley \& Cuthbert, 2008), which were divided in two versions. The selected photographs depicted 11 neutral objects (e.g. buildings tools) and 11 objects associated with contagion and contamination (e.g. cake with insects, a dirty toilet or garbage). Apart from their content, pictures were chosen based on their valence, arousal and disgust ratings known from past research (Lang, Bradley \& Cuthbert, 2008; Mikels et al., 2005). Thus, the „disgusting” images had average to high disgust ratings, average arousal and lower valence ratings. The neutral images had slightly positive valence ratings and low arousal and low disgust ratings. Every image would appear twice and for each one, the participants had to indicate on a visual analogue scale ranging from 0 to 100 , how disgusted they were by it, and as how dangerous they perceived it. This rather fine grained responding method was used to detect subtle changes.

To assess the changes in disgust sensitivity and danger perception between the different phases of the menstrual cycle, a repeated measures ANOVA was conducted, with menstrual phase (follicular vs. luteal phase) and image type (neutral vs. disgusting images) used as 
factors. To test the emotion recognition accuracy and the speed of labelling emotions as a function of the menstrual cycle, repeated measures ANOVA with menstrual cycle phase (follicular vs. luteal) and emotional expression (fear, anger, sadness, disgust, surprise, happiness) as factors was conducted.

\section{RESULTS}

For both, disgust and danger ratings, there was neither a significant main effect of the menstrual cycle phase $(\mathrm{F}(1,39)=0.566, \mathrm{p}=.457$ for disgust; $(\mathrm{F}(1,39)=0.020, \mathrm{p}=.887)$ for danger $)$, nor for the interaction between the phase and image type $(\mathrm{F}(1,39)=0.486, \mathrm{p}=$ .490 for disgust; $F(1,39)=0.015, \mathrm{p}=.902$ for danger). As can be seen on figures one and two, average disgust and danger ratings are very similar in both phases. Thus, the hypothesis was not validated. However, supporting the selected stimuli sets, pictures related to contagion and contamination were perceived as more disgusting $(\mathrm{F}(1,39)=399.607, \mathrm{p}=$ $.000)$ and dangerous $(F(1,39)=232.894, p=.000)$ than the neutral ones. The effect size, expressed as partial eta squared, is hp2 $=.911$ for disgust ratings, and hp2 $=.857$ for danger ratings.

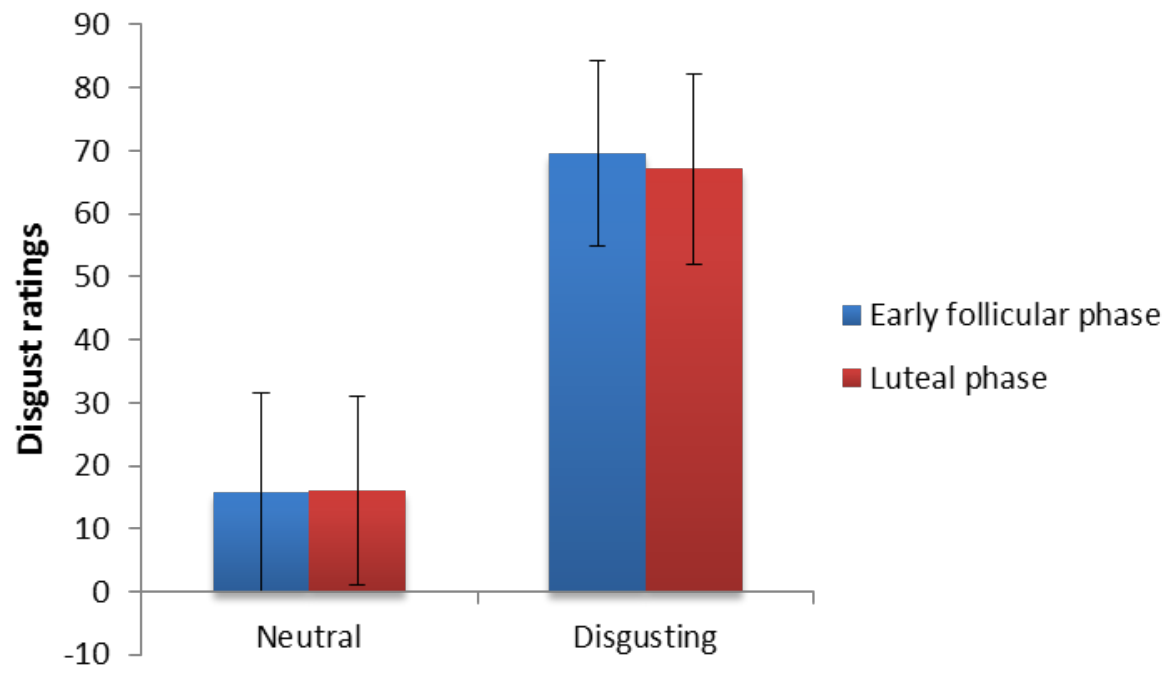

Figure 1. Average disgust ratings for neutral and "disgusting" images in the follicular and luteal phase of the cycle ( $\mathrm{N}=40$ participants) 


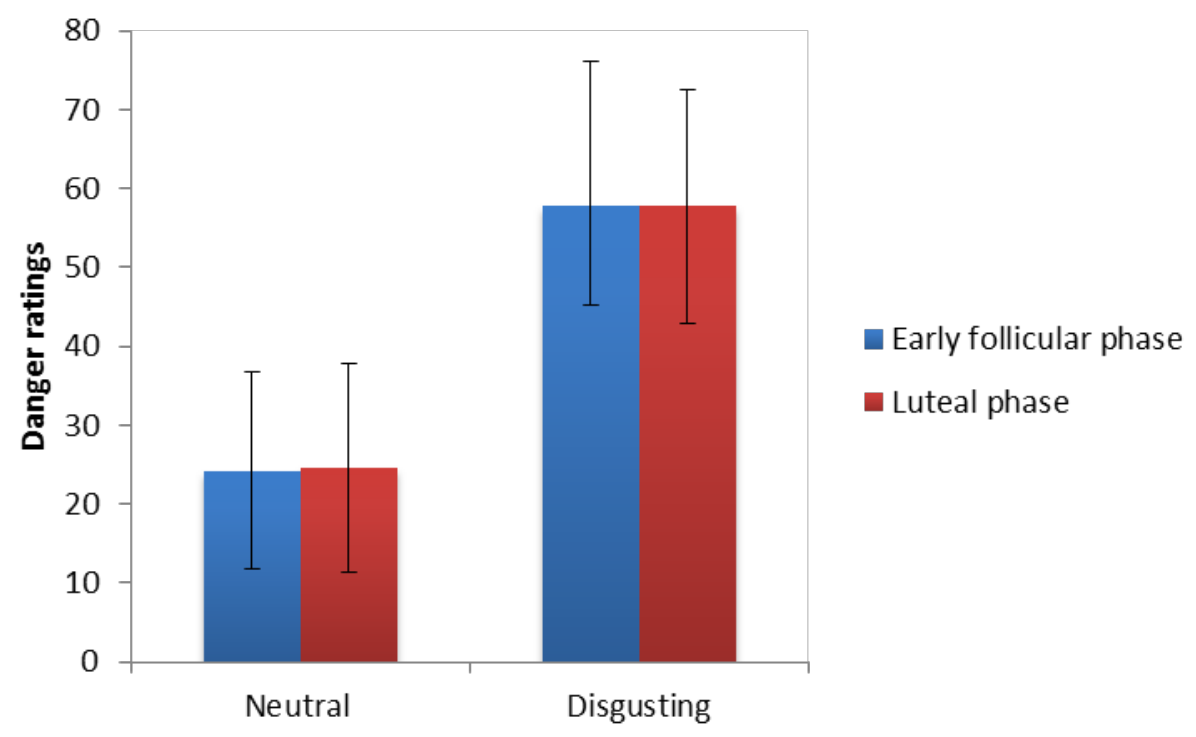

Figure 2. Average danger ratings for neutral and "disgusting" images in the follicular and luteal phase of the cycle ( $\mathrm{N}=40$ participants)

For the emotion recognition accuracy and labelling speed, a ANOVA was performed with Greenhouse-Geisser corrections due to violations of sphericity. The main effect of menstrual phase on emotion recognition was significant $(\mathrm{F}(1,39)=4.636, \mathrm{p}=.038)$, meaning a more accurate emotion recognition in the early follicular phase compared to the luteal, with the small effect size of $\eta_{\mathrm{p}}{ }^{2}=.106$. However, the interaction between the emotion and menstrual phase has not been found to be significant $(\mathrm{F}(4.339,169.221)=0.163, \mathrm{p}=$ .976). Therefore, contrary to expectations, the facial expression of disgust was not more accurately recognized in the luteal phase of the cycle, as can be observed on Figure 3 $(\mathrm{M}=.86, \mathrm{SD}=0.152$ in the early follicular phase; $\mathrm{M}=.83, \mathrm{SD}=0.164$ in the luteal phase). Moreover, the effect of emotion was significant $(F(3.065,119.550)=66.290, p=.000)$, with the effect size of $\eta_{\mathrm{p}}{ }^{2}=0.630$. A post-hoc Bonferroni correction showed that the fearful and sad expressions were less accurately recognized than other expressions.

Concerning the emotion labelling speed, both the main phase effect $(\mathrm{F}(1,39)=0.596, \mathrm{p}$ $=.445)$ and the phase $\mathrm{x}$ emotion interaction $(\mathrm{F}(3.990,155.593)=1.064, \mathrm{p}=.382)$ were statistically insignificant. The effect of emotion was significant $(F(3.516,137.119)=29.340$, $\mathrm{p}=.000)$, with the effect size of $\eta_{\mathrm{p}}^{2}=.429$, and the post-hoc Bonferroni showed slower labelling of the fearful and sad expressions compared to other expressions, and slower labelling of the surprised expression than the expression of happiness, as shown in figure 4. 
Mikolić, A.: Disgust and Facial Expression Recognition Across the Menstrual Cycle Human Ethology Bulletin - Proc. of the V. ISHE Summer Institute (2016): 60-73

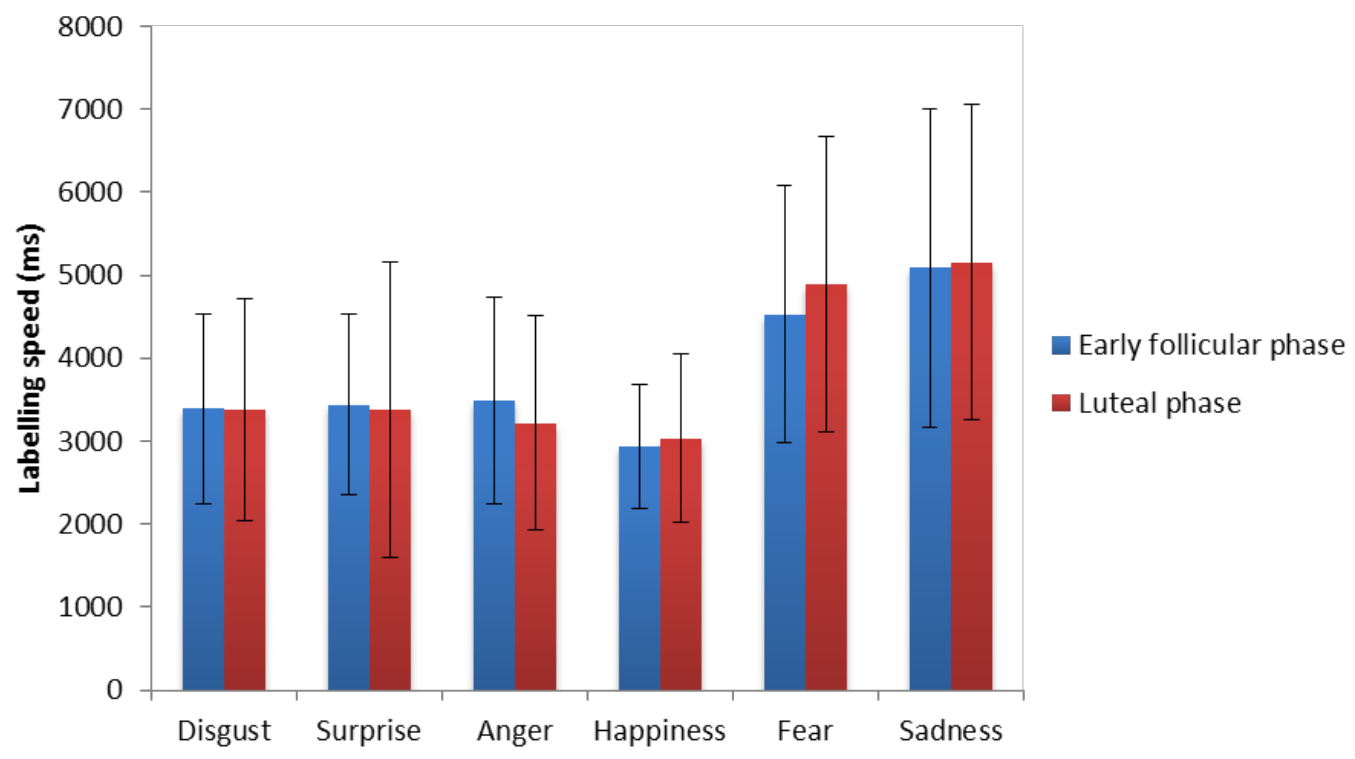

Figure 3. Average accuracy ratings for basic emotions (disgust, surprise, anger, happiness, fear, sadness $)$ in the follicular and luteal phases of the cycle ( $N=40$ participants $)$

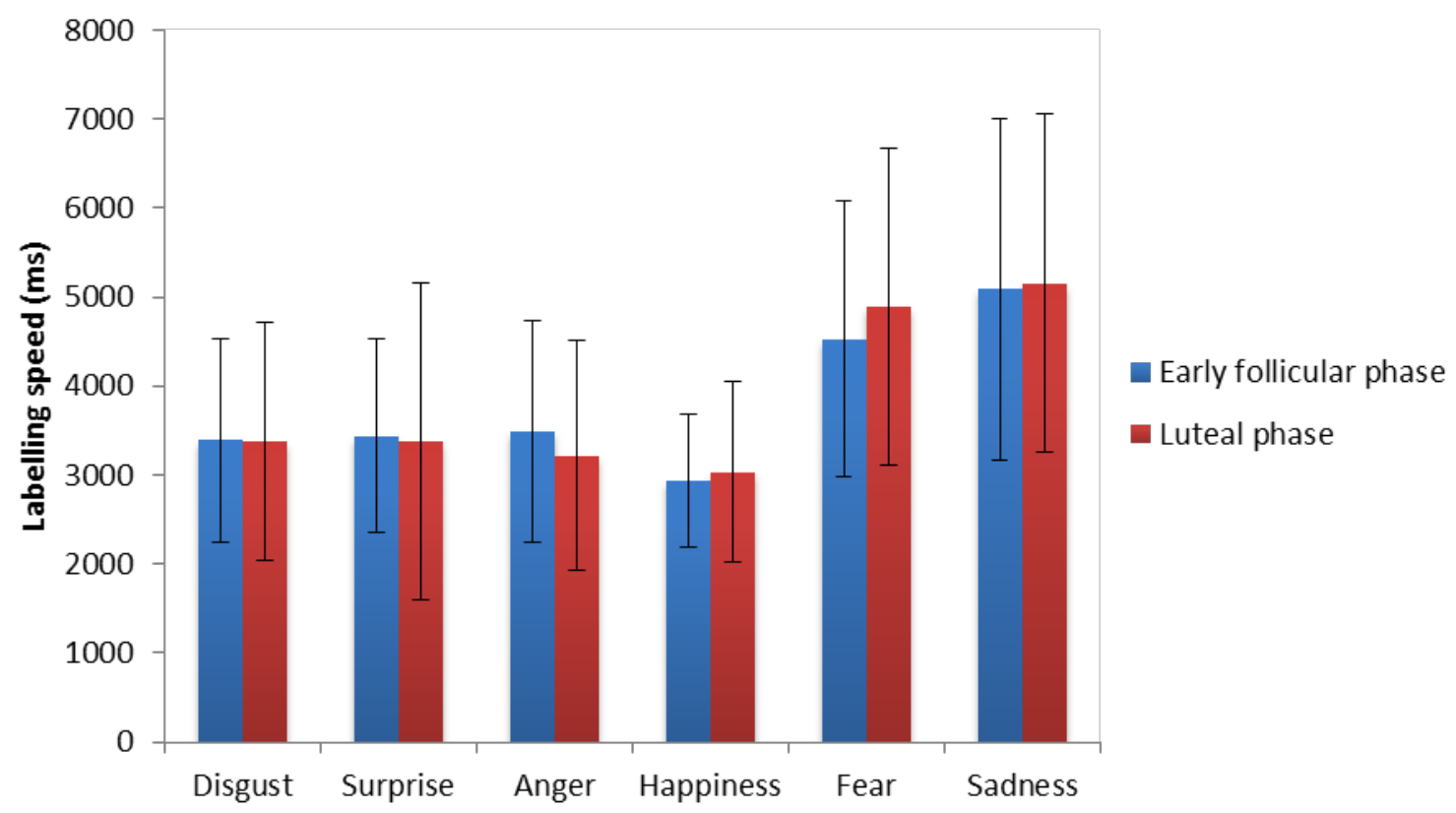

Figure 4. Average overall speed of labelling basic emotions (disgust, surprise, anger, happiness, fear, sadness) in the follicular and luteal phases of the cycle ( $\mathrm{N}=40$ participants $)$ 


\section{DISCUSSION}

The main goal of this study was to investigate compensatory prophylactic mechanisms in the luteal phase of the menstrual cycle. However, this hypothesis was not supported, since no expected changes in disgust sensitivity, perception of danger and recognition of expression of disgust have been observed. In addition, one of the hypotheses of this study was the change in emotion recognition accuracy across the menstrual cycle. Indeed, overall emotion recognition was more accurate in the early follicular phase compared to the luteal one. No changes in the speed of labelling emotions have been found as a function of the menstrual cycle. In the following the results of this study will be commented taking into account the methodological limitations and the results of previous research in the field.

Since the determination of menstrual phase was based on the dates of menses, and not on more precise methods for determining hormonal levels with blood or saliva analysis, this method might be the source of some inaccuracies. A study showed that the majority of menstrual cycles ( $77 \%$ ) differs from the average hormonal profile described in the literature (Alliende, 2002). Moreover, Fleischman and Fessler (2010) found a significant correlation between the levels of progesterone and disgust sensitivity, as well operationalized in terms of disgust ratings of photographs, but they assessed progesterone levels with saliva analysis. Moreover, the participants were not asked about their general and current health status, which could have influenced the progesterone levels and disgust sensitivity.

Another important limitation was the material used. The main effect of the image type has been found to be significant, showing that the photographs associated with contagion and contamination are indeed perceived as more disgusting and dangerous compared to the neutral ones. But, the question is if the selected disgusting pictures depict possible threats to our health well enough. Haidt et al. (1994) mention 8 domains of disgust, and most of them, except for the sexual and moral domain, were considered relevant for this study. Thereby, images of food (cake with insects), human fluids (vomit, faeces), body envelope violations (blood sampling, injured arm), animals (rat), hygiene (garbage) and death (animal corpse) were included. Nevertheless, perhaps the target images, directly associated with the infectious disease transmission, have still been underrepresented, such as spoiled foods, contagious cues (for example skin conditions) and contagious animals. Furthermore, although the two picture sets were rotated and counterbalanced among the participants and experimental conditions, the use of parallel versions would certainly improve the strength of conclusions in this study.

Regarding the recognition of the facial expression of disgust, it was assumed to be more accurate and faster in the luteal phase of the cycle. One of the possible reasons why this assumption has not been confirmed in this study is the stimulus presentation. Recent studies show that the meaning of facial expressions depends on the gaze direction (Adams \& Kleck, 2005; Jones et al., 2006). People seem to perceive fearful faces with averted gaze as more intense than faces with direct gaze (Adams \& Kleck, 2005). Accordingly, it can be assumed that the expression of disgust with averted gaze signals the cues of contagion in the environment, as opposed to the direct gaze which could indicate disgust with the observer., 
Women with higher levels of progesterone perceive expressions of disgust and fear with averted gaze as more intense in comparison to the faces with direct gaze (Conway et al., 2007). In this study, however, only expressions with direct gaze were used. In addition, expression of disgust had a high recognition accuracy $(\mathrm{M}=.86$ in the early follicular phase; $\mathrm{M}=.83$ in the luteal phase). Time limit or selection of different photographs could be used to make the task more challenging. Moreover, future research should be focused on the recognition errors women make in certain phases of the menstrual cycle. For instance, Derntl et al. (2009) found more errors in recognition of anger and disgust in the luteal group than in the follicular one. Overperceiving these emotions in the luteal phase is in line with the compensatory prophylactic hypothesis because it motivates avoidance instead of approaching, which facilitates protection from sources of health-threats.

Lastly, the presence of prophylactic compensatory behaviours in the luteal phase has not been consistently and clearly established yet. While Fleischman and Fessler (2010) have found changes in disgust sensitivity, bathroom behaviour, self-grooming and symptoms of obsessive-compulsive disorder as a function of progesterone, Fessler and Navarrette (2003) have not found heightened disgust in different hypothetical situations in periods of high progesterone. Therefore, further investigations are required, especially more ecologically valid studies with real-life situations and objects associated with cues of pathogens. It would be advisable to include relevant personality trait measures as well, such as perceived vulnerability to disease and neuroticism.

The main finding of this study is the more accurate emotion recognition of women in the early follicular phase compared to the luteal phase of the cycle. Changes in emotion recognition across the menstrual cycle have not been well investigated yet, but the obtained results are in line with the ones of Derntl et al. (2008). They observed better facial expressions recognition in the follicular phase than in the luteal phase, a negative correlation between levels of progesterone and recognition accuracy, and stronger activation of the amygdala in the follicular phase. Derntl et al. (2008) associate their results with the ones of Macrae et al. (2002), stating that women are more interested in social signals and interactions in the follicular phase, when they are more fertile and in a better mood, compared to the luteal phase. It seems that hormones facilitate more efficient social cognition when the risk of pregnancy is the highest (Macrae et al., 2002). Women in their follicular phase could be more focused on emotional expressions, which are the basis of successful social interactions, and in such a way increase their chances for reproduction (Derntl et al., 2008). In comparison the mood is more negative in the luteal phase, and mating is not of primary interest anymore. But, the study of Derntl et al. (2008) covered the whole follicular phase, from the beginning of the cycle until the ovulation. The question is if the mentioned explanation is equally relevant for the early follicular phase, which does not precede the ovulation. It may be that biased information processing and more negative mood in the luteal phase contribute to less accurate emotion recognition and specific errors in this period. 
Unlike recognition accuracy, speed has not been significantly different between the phases. It should be mentioned that this measure refers to the overall speed, and not just to the speed of correct answers. Moreover, factors such as reading speed and the order of options could influence the results as well.

\section{CONCLUSION}

The compensatory prophylactic hypothesis was not supported by this study: disgust sensitivity, perception of danger and the recognition accuracy of disgusted expression were not greater in the luteal phase of the menstrual cycle compared to the follicular. However, overall emotion recognition was more accurate in the follicular phase than in the luteal.

\section{REFERENCES}

Adams, Jr., R.B., Kleck, R.E. (2005). Effects of direct and averted gaze on the perception of facially communicated emotion. Emotion 5, 3-11. DOI

Alliende, Maria-Elena (2002). Mean versus individual hormonal profiles in the menstrual cycle. Fertility and Sterility, 78(1), 90-95. DOI

Conway, C.A., Jones, B.C., DeBruine, L.M., Welling, L.L.M., Law Smith, M.J., Perrett, D.I., Sharp, M.A., et al. (2007). Salience of emotional displays of danger and contagion in faces is enhanced when progesterone levels are raised. Hormones and Behavior, 51 (2), 202-206. DOI

Curtis, V., Aunger, R. \& Rabie, T. (2004). Evidence that disgust evolved to protect from risk of disease. Proceedings of the Royal Society 271(Suppl. 4), S131-S133. DOI

Curtis, V. \& Biran, A. (2001). Dirt, disgust, and disease: Is hygiene in our genes? Perspectives in Biology and Medicine, 44, 17-31. DOI

Curtis, V., de Barra, M., Aunger, R. (2011). Disgust as an adaptive system for disease avoidance behaviour. Philosophical Transactions of the Royal Society B: Biological Sciences, 366 (1563): 389-401. DOI

Derntl, B., Kryspin-Exner, I., Fernbach, E., Moser, E., \& Habel, U. (2008a). Emotion recognition accuracy in healthy young females is associated with cycle phase. Hormones and Behavior, 53(1), 90-95. DOI

Derntl, B., Windischberger, C., Robinson, S., Lamplmayr, E., Kryspin-Exner, I., Gur, R.C., et al. (2008). Facial emotion recognition andamygdala activation are associated with menstrual cycle phase. Psychoneuroendocrinology, 33, 1031-1040. DOI 
Mikolić, A.: Disgust and Facial Expression Recognition Across the Menstrual Cycle Human Ethology Bulletin - Proc. of the V. ISHE Summer Institute (2016): 60-73

Duncan, L.A., \& Schaller, M. (2009). Prejudicial attitudes toward older adults may be exaggerated when people feel vulnerable to infectious disease: Evidence and implications. Analyses of Social Issues and Public Policy, 9, 97-115. DOI

Ekman, P. \& Friesen, W.V. (1975). Unmasking the face. Englewood Cliffs, N.J.: Prentice-Hall.

Faas, M., Bouman, A., Moesa, H., Heineman, M.J., de Leij, L., Schuiling, G. (2000). The immune response during the luteal phase of the ovarian cycle: a Th2-type response? Fertility and Sterility. 74 (5), 1008-1013. DOI

Faulkner, J., Schaller, M., Park, J. H., \& Duncan, L. A. (2004). Evolved disease-avoidance mechanisms and contemporary xenophobic attitudes. Group Processes \& Intergroup Relations, 7 (4), 333-353. DOI

Fessler, D.M., Navarrete, C.D. (2003). Domain-specific variation in disgust sensitivity across the menstrual cycle. Evolution and Human. Behavior, 24 (6), 406-417. DOI

Fessler, D. M. T., Eng, S. J., \& Navarrete, C. D. (2005). Elevated disgust sensitivity in the first trimester: Evidence supporting the com- pensatory prophylaxis hypothesis. Evolution and Human Behavior, 26 (4), 344-351. DOI

Fleischman, D.S., Fessler, D.M.T. (2010). Progesterone's effects on the psychology of disease avoidance: Support for the compensatory behavioral prophylaxis hypothesis, Hormones and Behavior, 59 (2), 271-275. DOI

Fleischman, D.S., Fessler, D.M. (2007). Differences in dietary intake as a function of sexual activity and hormonal contraception. Evolutionary Psychology, 5(3), 642-652. DOI

Guapo, V. G., Graeff, F. G., Zani, A. C. T., Labate, C. M., dos Reis, R. M., \& Del-Ben, C. M. (2009). Effects of sex hormonal levels and phases of the menstrual cycle in the processing of emotional faces. Psychoneuroendocrinology, 34, 1087-1094. DOI

Haidt, J., McCauley, C., \& Rozin, P. (1994). Individual differences in sensitivity to disgust: A scale sampling seven domains of disgust elicitors. Personality and Individual Differences, 16, 701713. DOI

Jones, B.C., Perrett, D.I., Little, A.C., Boothroyd, L., Cornwell, R.E., Feinberg, D.R., Tiddeman, B.P., et al. (2005). Menstrual cycle, pregnancy and oral contraceptive use alter attraction to apparent health in faces. Proceedings of the Royal Society B: Biological Sciences, 272, (1561), 347. DOI

Lang, P.J., Bradley, M.M., \& Cuthbert, B.N. (2008). International affective picture system (IAPS): Affective ratings of pictures and instruction manual. Technical Report A-8. University of Florida, Gainesville, FL. 31

Macrae, C. N., Alnwick, K. A., Milne, A. B., \& Schloerscheidt, A. M. (2002). Person perception across the menstrual cycle: Hormonal influences on social-cognitive functioning. Psychological Science, 13(6), 532-536. DOI

McFarlane, J. Martin, C.L. Williams, T.M. (1988). Mood fluctuations: Women versus men and menstrual versus other cycles. Psychology of Women Quarterly, 12, 201-233. DOI 
Mikolić, A.: Disgust and Facial Expression Recognition Across the Menstrual Cycle Human Ethology Bulletin - Proc. of the V. ISHE Summer Institute (2016): 60-73

Mikels, J. A., Fredrickson, B. L., Larkin, G.R., Lindberg, C.M., Maglio, S.J., \& Reuter-Lorenz, P.A. (2005). Emotional category data on images from the International Affective Picture System. Behavior Research Methods, 37, 626-630. DOI

Mortensen, C.R., Becker, D.V., Ackerman, J.M., Neuberg, S.L., \& Kenrick, D.T. (2010). Infection breeds reticence: The effects of disease salience on self-perceptions of personality and behavioral tendencies. Psychological Science, 21, 440-447. DOI

Navarrete, C. D., \& Fessler, D. M. T. (2006). Disease avoidance and ethnocentrism: The effects of disease vulnerability and disgust sensitivity on intergroup attitudes. Evolution and Human Behavior, 27, 270-282. DOI

Park, J. H., Faulkner, J., \& Schaller, M. (2003). Evolved disease-avoidance processes and contemporary anti-social behavior: Prejudicial attitudes and avoidance of people with physical disabilities. Journal of Nonverbal Behavior, 27, 65-87. DOI

Park, J. H., Schaller, M., \& Crandall, C. S. (2007). Pathogen-avoidance mechanisms and the stigmatization of obese people. Evolution and Human Behavior, 28, 410-414. DOI

Prokop, P., Uşak, M., Fančovičová, J. (2010). Health and the avoidance of macroparasites: A preliminary cross-cultural study. Journal of Ethology, 28(2), 345 - 351. DOI

Schaller, M., \& Duncan, L. A. (2007). The behavioral immune system: Its evolution and social psychological implications. In J.P. Forgas, M.G. Haselton, \& W. von Hippel 32 (Eds.), Evolution and the social mind: Evolutionary psychology and social cognition (pp. 293-307). New York: Psychology Press.

Schaller, M., Park, J.H., \& Kenrick, D T. (2007). Human evolution and social cognition. In R.I.M. Dunbar \& L. Barrett (Eds.), Oxford handbook of evolutionary psychology. (pp. 491-504). Oxford, England: Oxford University Press. DOI

Schneider, W., Eschman, A., \& Zuccolotto, A. (2002). Prime user's guide. Pittsburgh, PA: Psychology Software Tools, Inc.

Tottenham, N., Tanaka, J., Leon, A.C., McCarry, T., Nurse, M., Hare, T.A., Marcus, D.J., Westerlund, A., Casey, B.J., Nelson, C.A. (2009). The NimStim set of facial expressions: judgments from untrained research participants. Psychiatry Research, 168(3):242-9. DOI

Trzonkowski, P., Myśliwska, J., Tukaszuk, K., Szmit, E., Bryl, E., Myśliwski, A. (2001). Luteal phase of the menstrual cycle in young healthy women is associated with decline in interleukin 2 levels. Hormone and Metabolic Research, 33(6), 348-353. DOI

Van Wingen, G.A., van Broekhoven, F., Verkes, R.J., Petersson, K.M., Bäckström, T., Buitelaar, J.K., Fernandez, G., (2007a). Progesterone selectively increases amygdala reactivity in women. Molecular Psychiatry, 13, 325-333. DOI

Welling, L. L. M., Conway C. A., Debruine L. M., Jones, B. C. (2007). Perceived vulnerability to disease is positively related to the strength of preferences for apparent health in faces. Journal of Evolutionary Psychology, 5(1-4), 131-139. DOI 